\title{
EDITORIAL
}

\section{Is difficult asthma still clinically meaningful?}

\author{
P. Chanez and P. Godard
}

$\Delta$ sthma is still a clinically prevalent and important chronic disease worldwide. A wide range of efficacious asthma medications and numerous national and international recommendations for their usage are now available. Thus, it is often a low rate of patient adherence and severity of the disease that pose the major challenge to effective asthma management. Physicians have learnt to treat asthmatics in order to achieve the best optimal control, to decrease mortality and prevent lung function decline, whereas patients are usually more concerned with their ability to live normally without any chronic disease and without any potential deleterious effects from therapy [1].

In 1999, a European Respiratory Society (ERS) Task Force adopted the term "difficult asthma", which was defined as "asthma, poorly controlled in terms of chronic symptoms, with episodic exacerbations, persistent and variable airway obstruction and continued requirement for short-acting $\beta_{2}$-agonists and a reasonable dose of inhaled corticosteroids" [2]. These symptoms occur in a minority of patients with asthma, but they represent the highest consumers of health services, and their daily lives are severely affected by their chronic disease [3]. A potential contributor to poor patient adherence to therapy may be a discrepancy between the aims of the doctor and those of the patient. This concept of difficult asthma challenges the current views on asthma as a benign, easy to diagnose and treat disease and requires specific extensive re-evaluation including diagnosis, management and careful long-term specialised follow-up.

We propose that the term "difficult asthma" is clinically very meaningful and that it should encourage patients to undergo a rigorous and systematic approach in a specialised centre using a multidisciplinary team. There have been several reports showing that this procedure is of benefit; however, further evaluations are certainly needed [4]. We believe that the diagnosis of severe asthma should be reserved for those who have refractory asthma after extensive re-evaluation and an appropriate observation period of at least 6 months [5]. This is not trivial because it will help to assess the validity of the correct diagnosis of asthma, the importance of triggers and comorbid conditions, and finally the contribution of poor adherence to the poor control of the disease. This rigorous approach is the only way to improve our understanding of the complexity of severe asthma, define new phenotypes and test new treatments with a real potential for success.

Hopital Arnaud de Villeneuve, Clinique des Maladies Respiratories, Montpellier, France.

CORRESPONDENCE: P. Chanez, Clinique des Maladies Respiratoires, 34295 Montpellier, Cedex 5, France. Fax: 33 467521848. E-mail: chanez@montp.inserm.fr

\section{IS ASTHMA THE CORRECT DIAGNOSIS?}

Most of the definitions of asthma are descriptive in nature. The two characteristic elements are clinical (chronicity, variability and reversibility of symptoms) and functional. There have been several studies on the sensitivity and specificity of the functional signs of asthma and their predictive values are clearly insufficient. Those maintained in the international recommendations are cough, dyspnoea, wheezing, chest tightness and sputum production. However, most physicians, especially in primary care, are confident in their ability to make an easy diagnosis of this disease. Definition of reversibility or its significance is only obtained from expert opinions. The same is true for the usefulness of the corticosteroid test to identify asthmatic patients: a short course of oral corticosteroid is generally used. The dose and duration of steroid treatment given in the literature are highly variable. However, these criteria are generally considered to be sufficient to propose the diagnosis of asthma and to potentially qualify nonresponding asthmatic patients as steroid-resistant [6]. In a patient referred as having difficult asthma, the diagnosis of severe asthma is predicated by a secure diagnosis of asthma. For this reason, it must be confirmed by the presence of typical symptoms, together with objective evidence of variable airflow limitation and/or airway hyperresponsiveness. Demonstration of reversible airways obstruction by short-acting bronchodilators and/ or corticosteroids is valuable, but bronchial provocation testing is more sensitive and specific and should be performed when necessary [7]. However, when airflow impairment is present, bronchial provocation may not be helpful and/or feasible from a safety and regulatory perspective [8].

In addition, alternative diagnoses need to be considered and excluded. This is not always an easy task; chronic obstructive pulmonary disease (COPD) is an example. A recent editorial demonstrated that potential combinations between asthma and COPD may exist, which can vary in time and from one patient to another [9]. Furthermore, vocal cord dysfunction (VCD) is frequently misdiagnosed as difficult asthma. These conditions require specific expert diagnosis and management. The major problems are represented by the absence of clear diagnosis guidelines and the coexistence of real asthma in conjunction with VCD in many cases.

\section{ARE SPECIFIC TRIGGERS STILL PRESENT AND DELETERIOUS?}

Ongoing allergen exposure or occupational agent may contribute to the poor control of asthma, although, in adults, allergic responses have not been considered to be among the strongest contributing factors to asthma severity [10]. The direct contribution and clinical relevance of allergenic persistent exposition is difficult to establish in real life and deserves a 
follow-up period integrating objective measures. Asthmatic smokers received recent attention and are often difficult asthmatics. However, a recent large study did not find a relationship between smoking and severity or accelerated decline in forced expiratory volume in one second [11]. Specific attention to the diagnosis and response to treatment of these patients is needed as they usually do not respond to steroids [12].

\section{WHAT IS THE CONTRIBUTION OF COMORBID CONDITIONS?}

First, the definition of a comorbid condition is not an easy task. Some comorbid conditions, such as rhinosinusitis, should be integrated to the "asthma definition" due to their high frequency [13]. They should always be treated as they have been shown to influence asthma control on several occasions. Other factors [13] have been associated with difficult asthma, gastro-oesophageal reflux (GOR) [14], recurrent respiratory infections, psychological dysfunction and obstructive sleep apnoea [15]. The role of GOR disease in difficult asthma is controversial. The frequent lack of improvement with treatment suggests that its contribution to asthma control is variable. Obesity is increasingly prevalent and has been associated with difficult asthma. Weight reduction is associated with better asthma control when bronchial hyperreactivity is not affected. The correlation between obesity and difficult asthma is more frequently reported in females. The role of different factors, including hormonal influences and the perception of symptoms, are of paramount importance to better understand and treat asthma in overweight patients [16]. Depression, anxiety and behavioural problems may be detrimental to asthma control but also may be a consequence of it. There is no clear psychological profile associated with difficult asthma, although common psychological diseases, such as depression and anxiety, are more common in severe asthma, particularly in association with near-fatal asthma. There are some psychological features associated with difficult asthma and they should be investigated and discussed with the patient. However, the more severe forms of psychological problems have not been associated with severe asthma compared with milder disease [17]. The recognition of comorbidities is an essential component of the assessment of difficult asthma, the impact of managing these comorbid conditions on asthma outcomes remains to be fully addressed.

\section{Adherence to treatment}

Adherence is often suboptimal in chronic diseases and it is well established in asthma. Poor asthma control has been associated with insufficient corticosteroid treatment adherence, and lack of adherence is one of the main factors linked to acute exacerbations. Lack of adherence is a potential contributor to difficult asthma and may increase the perceived severity of the disease by both doctors and patients [18]. Adherence represents a critical issue, but how to best assess and manage poor adherence remains a significant challenge. Electronic monitoring seems ideal although not easily available. Of note, adherence may vary according to disease severity, with the most severe patients being the most adherent to management. Adherence is certainly multifactorial, variable in time and from one patient to another. Psychological factors may affect adherence but the relationships are certainly complex and deserve, once again, a long-term follow-up period.

Clinical evaluation of difficult asthma should be made in specialised centres. Specific investigations are helpful including: high-resolution computed tomography (CT) of the chest; ear, nose and throat examination and sinus $\mathrm{CT}$; $\mathrm{pH}$ probe to evaluate GOR disease; allergen skin testing; full sleep study; full pulmonary function tests; formal psychological assessment; and evaluation of treatment adherence. This approach is time consuming, requires various experts with specific knowledge and should be repeated over a 6-month to 1-yr period to assess potential changes (fig. 1).

\section{MANAGEMENT OF DIFFICULT ASTHMA IN DAILY PRACTICE}

In the present issue of the European Respiratory Journal (ERJ), ROBERTS et al. [19] question the clinical management of difficult asthma in the UK. A postal survey was mailed to 683 consultant members of the British Thoracic Society, outlining five cases of difficult asthma and eliciting responses to a range of questions concerning investigations, consultation of other health professionals, differential diagnoses, misdiagnoses, and differences between specialists with and without a particular interest in difficult asthma. The study by ROBERTS et al. [19] is useful, particularly in making the important distinction between severe asthma and difficult asthma. It also outlines the important role of misdiagnoses and coexisting psychosocial

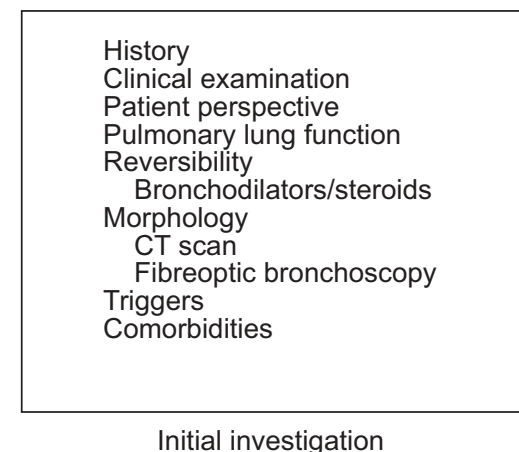

Initial investigation

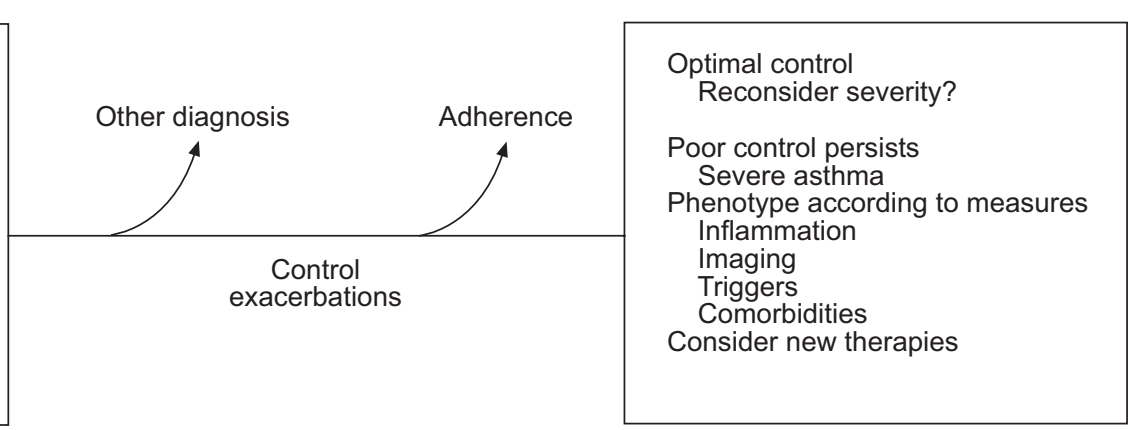

Second investigation 
issues, which prevent good asthma outcomes despite maximal therapy. The study also highlights the potentially important impact of failing to assess compliance or access liaison psychiatric review. It is clear from the survey that poor availability of support from psychiatric and psychological services is a major issue. The limitations of the study by ROBERTS et al. [19] are obviously the size of the survey and its response rate. However, the data are based on a self report from healthcare providers, but can be representative of the situation for difficult asthma in a European country at a given time. It may provide a basis to establish national and European networks to better understand difficult asthma.

In conclusion, the observational study in the current issue of the ERJ [19], despite its limitations, reinforces our strong beliefs that "difficult asthma" is still clinically meaningful. It pleads for a better use of the guideline issues from the ERS Task Force, and for establishing and publicising centralised centres with specific interest and expertise within Europe. The power of phenotyping severe asthma will be increased manifold by establishing severe asthma networks, such as the existing ENFUMOSA (European Network for Understanding Mechanisms of Severe Asthma) [20] and BIOAIR (Longitudinal Assessment of Clinical Course and BIOmarkers in Severe Chronic AIRway Disease) in Europe and the National Heart Lung and Blood Institute network in the USA, which will allow the enrolment of more patients.

We believe that a clear approach to objectively diagnose and assess difficult asthma will provide an insight into the real prevalence of severe asthma. Network and cohort follow-up should be encouraged to standardise the diagnosis and assessment of severe asthma using a common panel of outcome measures. Cross-sectional and longitudinal investigations can be captured to provide a better opportunity to unravel the complex heterogeneity of severe asthma leading to improved outcomes [21].

\section{REFERENCES}

1 Reddel H. Goals of asthma treatment: how high should we go? Eur Respir J 2004; 24: 15-17.

2 Chung K, Godard P, Adelroth E, et al. Difficult/therapy resistant asthma: the need for an integrated approach to define clinical phenotypes, evaluate risk factors, understand pathophysiology and find novel therapies. ERS Task Force on Difficult/Therapy-Resistant Asthma. Eur Respir J 1999; 13: 1198-1208.

3 Godard P, Chanez P, Siraudin L, Nicoloyannis N, Duru G. Costs of asthma are correlated with severity: a 1-yr prospective study. Eur Respir J 2002; 19: 61-67.
4 Robinson DS, Campbell DA, Durham SR, Pfeffer J, Barnes PJ, Chung KF. Systematic assessment of difficultto-treat asthma. Eur Respir J 2003; 22: 478-483.

5 Proceedings of the ATS workshop on refractory asthma. current understanding, recommendations, and unanswered questions. American Thoracic Society. Am J Respir Crit Care Med 2000; 162: 2341-2351.

6 Barnes PJ. Corticosteroid resistance in airway disease. Proc Am Thorac Soc 2004; 1: 264-268.

7 Hunter CJ, Brightling CE, Woltmann G, Wardlaw AJ, Pavord ID. A comparison of the validity of different diagnostic tests in adults with asthma. Chest 2002; 121: 1051-1057.

8 Wenzel SE. Severe asthma in adults. Exp Lung Res 2005; 31: Suppl. 1, 22.

9 Hargreave FE, Parameswaran K. Asthma, COPD and bronchitis are just components of airway disease. Eur Respir J 2006; 28: 264-267.

10 Miranda C, Busacker A, Balzar S, Trudeau J, Wenzel SE. Distinguishing severe asthma phenotypes: role of age at onset and eosinophilic inflammation. I Allergy Clin Immunol 2004; 113: 101-108.

11 de Marco R, Marcon A, Jarvis D, et al. Prognostic factors of asthma severity: a 9-year international prospective cohort study. J Allergy Clin Immunol 2006; 117: 1249-1256.

12 Thomson NC, Chaudhuri R, Livingston E. Asthma and cigarette smoking. Eur Respir J 2004; 24: 822-833.

13 ten Brinke A, Sterk PJ, Masclee AA, et al. Risk factors of frequent exacerbations in difficult-to-treat asthma. Eur Respir J 2005; 26: 812-818.

14 Harding SM. Gastroesophageal reflux: a potential asthma trigger. Immunol Allergy Clin North Am 2005; 25: 131-148.

15 Yigla M, Tov N, Solomonov A, Rubin AH, Harlev D. Difficult-to-control asthma and obstructive sleep apnea. J Asthma 2003; 40: 865-871.

16 Shore SA, Johnston RA. Obesity and asthma. Pharmacol Ther 2006; 110: 83-102.

17 ten Brinke A, Ouwerkerk ME, Bel EH, Spinhoven P. Similar psychological characteristics in mild and severe asthma. J Psychosom Res 2001; 50: 7-10.

18 Lacasse Y, Archibald H, Ernst P, Boulet LP. Patterns and determinants of compliance with inhaled steroids in adults with asthma. Can Respir J 2005; 12: 211-217.

19 Roberts NJ, Robinson DS, Partridge MR. How is difficult asthma managed? Eur Respir J 2006; 28: 968-973.

20 The ENFUMOSA cross-sectional European multicentre study of the clinical phenotype of chronic severe asthma. European Network for Understanding Mechanisms of Severe Asthma. Eur Respir J 2003; 22: 470-477.

21 Bel EH. Clinical phenotypes of asthma. Curr Opin Pulm Med 2004; 10: 44-50. 